\title{
Long non-coding RNA TUG1 promotes osteosarcoma cell proliferation and invasion through inhibition of microRNA-212-3p expression
}

\author{
HENG LI*, GUOFENG TIAN*, FEIPENG TIAN and LIN SHAO \\ Department of Orthopedics, Daqing Longnan Hospital, Daqing, Heilongjiang 163453, P.R. China
}

Received January 2, 2018; Accepted April 20, 2018

DOI: $10.3892 /$ etm.2018.6216

\begin{abstract}
Taurine upregulated gene 1 (TUG1), a long non-coding RNA (lncRNA), has recently been suggested to be associated with the development of osteosarcoma (OS), but the underlying molecular mechanism still remains largely unclear. In the present study, it was revealed that TUG1 was significantly upregulated whereas miR-212-3p was significantly downregulated in OS tissues and cell lines, when compared with adjacent non-tumor tissues and normal osteoblasts cell lines, respectively. An inverse association between the TUG1 and miR-212-3p expression was also observed in OS tissues. Furthermore, TUG1 directly interacted with miR-212-3p and negatively regulated the expression of miR-212-3p in OS cells. In vitro experiments further indicated that inhibition of TUG1 suppressed the proliferation and invasion of OS cells. Furthermore, knockdown of miR-212-3p eliminated the suppressive effects of TUG1 inhibition on the proliferation and invasion of OS cells. Taken together, these findings demonstrate that TUG1 promotes OS cell proliferation and invasion by inhibition of miR-212-3p expression, thus suggesting that TUG1 may become a potential therapeutic target for OS.
\end{abstract}

\section{Introduction}

Osteosarcoma (OS) is the most prevailing primary malignant tumor in the bone $(1,2)$. Although advances have been made on the treatment of OS including neoadjuvant or adjuvant chemotherapy combined with surgery and radiotherapy, the 5-year survival rate of patients with advanced OS has not significantly improved from $27.4 \%$ (2-4). Exploring the molecular mechanisms underlying the development and progression of

Correspondence to: Professor Lin Shao, Department of Orthopedics, Daqing Longnan Hospital, 35 Aiguo Road, Daqing, Heilongjiang 163453, P.R. China

E-mail: daqingshaolin@sina.com

\section{*Contributed equally}

Key words: long non-coding RNA, microRNA, osteosarcoma, proliferation, migration
OS is beneficial for identifying novel and effective therapeutic targets for this disease (2).

Long non-coding RNAs (IncRNAs), a class of regulatory transcripts $>200$ nucleotides, function mainly through interaction with mRNAs, microRNAs (miRNAs or miRs) or proteins $(5,6)$. miRNAs, a class of non-coding small RNAs containing 22-25 nucleotides, are also key regulators for gene expression via directly binding to the 3'-untranslated region of their target mRNAs, causing mRNA degradation or translation repression $(7,8)$. It has been established that both lncRNAs and miRNAs participate in a variety of cellular biological processes including cell proliferation, apoptosis, differentiation, motility and tumorigenesis (9-11). Furthermore, a large number of lncRNAs and miRNAs are deregulated in human cancers, and many have been identified to act as oncogenes or tumor suppressors (12-14).

The lncRNA taurine upregulated gene 1 (TUG1) is located at chr22q12.2 and has been reported to be deregulated in some common human cancers, and generally serve an oncogenic role (15). For instance, TUG1 promotes cervical cancer progression by regulating the miR-138-5p-Sirtuin 1 axis (16). Yan et al (17) recently reported that TUG1 promoted the malignant progression of oral squamous cell carcinoma through upregulating formin-like protein 2 by directly binding to miR-219. Recently, TUG1 was reported to be aberrantly overexpressed in OS, and its upregulation correlated with distant metastasis as well as poor prognosis of patients with OS (18). Furthermore, TUG1 has also been demonstrated to sponge several miRNAs to serve its promoting role in OS, including miR-9, miR-144, miR-153, and miR-335 (19-22). For instance, Wang et al (21) recently reported that knockdown of TUG1 inhibits OS cell proliferation and invasion by sponging miR-153. In addition, TUG1 promotes OS cell migration and invasion by acting as a competing endogenous RNA (ceRNA) of miR-335-5p (20). However, whether other miRNAs are also sponged by TUG1 in OS cells remains unclear.

miR-212 has been demonstrated to generally act as a tumor suppressor in certain common human cancers. For instance, miR-212 is downregulated and suppresses methyl-CpG-binding protein in human gastric cancer (23). A number of previous studies have reported that miR-212 has a suppressive role in OS cell proliferation and invasion via inhibiting the expression levels of their target genes such as SRY-box 4 (SOX4) and forkhead box A1 (FOXA1) $(24,25)$. However, to the best of our 
knowledge the association between TUG1 and miR-212 in OS has not previously been reported.

The present study aimed to explore the regulatory mechanism of TUG1 underlying OS cell proliferation and invasion in vitro.

\section{Materials and methods}

Clinical tissues. The present study was approved by the Medical Ethics Committee of Daqing Longnan Hospital (Daqing, China). A total of 21 OS tissues and matched adjacent non-tumor tissues were collected from patients with OS during surgical resection at Longnan Hospital (Daqing, China) between March 2014 and September 2016. These 21 patients included 14 males and 7 females, aged between 11 and 26 years (mean, 17.8 years). None of the patients had tumor history or had received radiochemotherapy prior to surgery. All patients provided written informed consent. The tissues were immediately frozen in liquid nitrogen and stored at $-80^{\circ} \mathrm{C}$ prior to further use.

Cell culture. Human osteoblast cell line HFOB1.19 and several common human OS cell lines including Saos-2, U2OS, HOS and MG63 were purchased from the Cell Bank of Chinese Academy of Sciences, Shanghai, China. All cell lines were cultured in Dulbecco's modified Eagle's medium (DMEM; Thermo Fisher Scientific, Inc., Waltham, MA, USA) supplemented with $10 \%$ fetal bovine serum (FBS; Thermo Fisher Scientific, Inc.). Cells were incubated at $37^{\circ} \mathrm{C}$ in a humidifiec atmosphere with $5 \% \mathrm{CO}_{2}$.

Cell transfection. Lipofectamine 2000 (Thermo Fisher Scientific, Inc.) was used to transfect Saos-2 and U2OS cells with $100 \mathrm{nM}$ non-specific short interfering RNA (siRNA; NC siRNA; cat. no. sc-37007; Santa Cruz Biotechnology, Inc., Dallas, TX, USA), 100 nM TUG1-specific siRNA (TUG1 siRNA; cat. no. YB2421; Yearthbio, Changsha, China), $100 \mathrm{nM}$ pcDNA3.1 vector, $100 \mathrm{nM}$ pcDNA-TUG1 expression plasmid (cat. no. YB1317; Yearthbio), $100 \mathrm{nM}$ miR-212-3p inhibitor (cat. no. HmiR-AN0319-SN-10; Guangzhou Fulengen Co., Ltd., Guangzhou, China), and $100 \mathrm{nM}$ negative control (NC) inhibitor (cat. no. CmiR-AN0001-SN; Guangzhou Fulengen Co., Ltd.), respectively, according to the manufacturer's instruction. Following transfection for $48 \mathrm{~h}$, experiments were performed.

Reverse transcription-quantitative polymerase chain reaction (RT-qPCR) analysis. Total RNA was isolated from clinical tissues and cells using TRIzol reagent (Thermo Fisher Scientific, Inc.), and then reverse transcribed into cDNA using a RevertAid First Stand cDNA Synthesis kit (Thermo Fisher Scientific, Inc.), according to the manufacturer's protocol. A SYBR ${ }^{\circledR}$ Premix Ex Taq ${ }^{\mathrm{TM}}$ Tli RNaseH Plus PCR kit (Takara Biotechnology Co., Ltd., Dalian, China) was used to examine the expression of TUG1 with an ABI 7300 Sequence Detector (Thermo Fisher Scientific, Inc.). A TaqMan Human miRNA assay kit (Thermo Fisher Scientific, Inc.) was used to examine the miR-212-3p expression. GAPDH and U6 were used as the internal references. The reaction conditions were $95^{\circ} \mathrm{C}$ for $3 \mathrm{~min}$, followed by 40 cycles of $95^{\circ} \mathrm{C}$ for $15 \mathrm{sec}$ and $60^{\circ} \mathrm{C}$ for $60 \mathrm{sec}$. The relative gene expression was calculated using the $2^{-\triangle \Delta C q}$ method (26). The primers sequences were as follows: TUG1, forward 5'-CTGAAGAAAGGCAACATC-3' and reverse 5'-GTAGGCTACTACAGGATTTG-3'; GAPDH, forward 5'-GGAGCGAGATCCCTCCAAAAT-3' and reverse 5'-GGCTGTTGTCATACTTCTCATGG-3'; miR-212-3p, forward 5'-GGTAACAGTCTCCAGTCA-3' and reverse 5'-GCAATTGCACTGGATACG-3'; and U6, forward 5'-CTC GCTTCGGCAGCACATATACT-3' and reverse 5'-CGCTTC ACGAATTTGCGTGT-3'.

Western blotting. Cells were lysed in radioimmunoprecipitation assay buffer (Thermo Fisher Scientific, Inc.) containing protease inhibitor (Thermo Fisher Scientific, Inc.). The protein concentration was determined using a bicinchoninic acid protein assay kit (Beyotime Institute of Biotechnology, Haimen, China), according to the manufacturer's protocol. Protein samples $(50 \mu \mathrm{g})$ were separated by $12 \%$ SDS-PAGE, and then transferred to a polyyinylidene difluoride membrane (EMD Millipore, Billerica, MA, USA). The membrane was then blocked in 5\% non-fat dried milk in TBS with 0.1\% Tween-20 (Sigma-Aldrich; Merck KGaA, Darmstadt, Germany) at room temperature for $3 \mathrm{~h}$. Subsequently, the membrane was incubated with rabbit anti-matrix metalloproteinase (MMP)2 (1:50; cat. no. ab37150), anti-MMP9 (1:50; cat. no. ab38898) and anti-GAPDH (1:100; cat. no. ab9485; all Abcam, Cambridge, UK) primary antibodies at room temperature for $3 \mathrm{~h}$. Following washing with PBS with Tween-20 3 times, the membrane was incubated with a horseradish peroxidase-conjugated secondary antibody $(1: 5,000$; cat. no. ab6721; Abcam) at room temperature for $40 \mathrm{~min}$. The protein signal was detected using the enhanced chemiluminescence reagents (Pierce; Thermo Fisher Scientific, Inc.), and the densitometric analysis was conducted using ImageJ software 1.48 (National Institutes of Health, Bethesda, MD, USA).

Cell Counting Kit-8 (CCK-8) assay. Transfected cells $\left(1.5 \times 10^{3}\right.$ per well $)$ were plated in 96 -well culture plates, and cultured for 0, 24, 48 and $72 \mathrm{~h}$. Cell proliferation was determined using CCK-8 (Dojindo Molecular Technologies, Inc., Kumamoto, Japan), according to the manufacturer's protocol. Absorbance was detected at $450 \mathrm{~nm}$ on a microplate reader (Molecular Devices, LLC, Sunnyvale, CA, USA).

Cell invasion assay. A total of $1 \times 10^{5}$ cells were seeded into the upper chamber of a Transwell plate pre-coated with Matrigel (HyClone; GE Healthcare Life Sciences, Logan, UT, USA) containing $200 \mu \mathrm{l}$ serum-free DMEM, and $500 \mu \mathrm{l}$ DMEM with 10\% FBS was added into the lower chamber. Following incubation at $37^{\circ} \mathrm{C}$ for $24 \mathrm{~h}$, the remaining cells in the upper chamber were discarded, and the membrane was fixed with $4 \%$ formaldehyde for $20 \mathrm{~min}$ and stained with Giemsa for $10 \mathrm{~min}$ at room temperature. The invasive cells were observed and counted under a light microscope (magnification, $\mathrm{x} 400$ ).

Bioinformatics analysis. The target genes for TUG1 and miR-212-3p were predicted using RNAhybrid 2.12 (http://bibiserv.techfak.uni-bielefeld.de/rnahybrid/). 

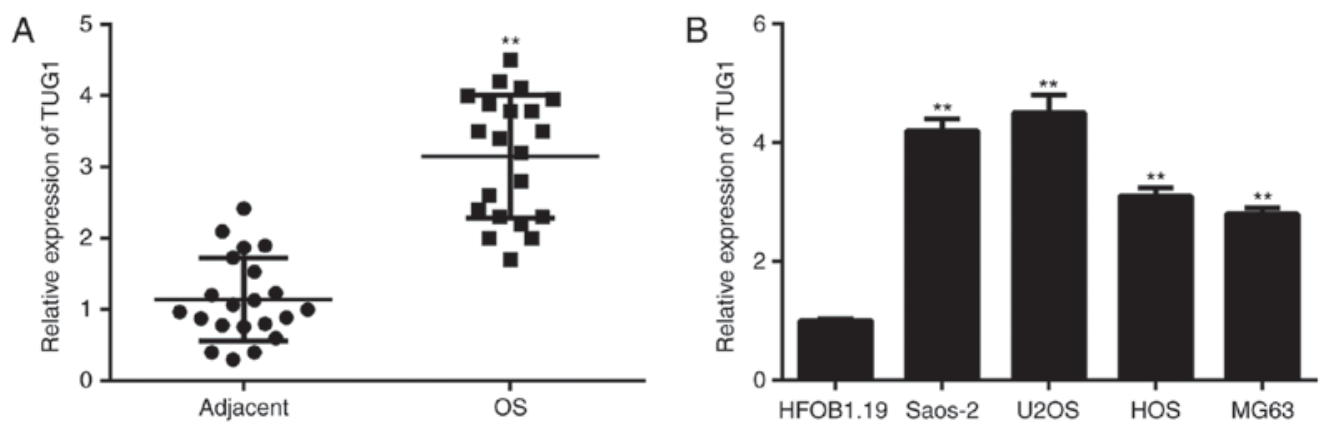

Figure 1. (A) RT-qPCR was conducted to examine the expression of TUG1 in OS tissues compared with adjacent non-tumor tissues. ** $\mathrm{P}<0.01$ vs. adjacent. (B) RT-qPCR was conducted to examine the expression of TUG1 in OS cell lines compared with normal human osteoblast cell line HFOB1.19. ${ }^{* *} \mathrm{P}<0.01$ vs. HFOB1.19. RT-qPCR, reverse transcription-quantitative polymerase chain reaction; TUG1, taurine upregulated gene 1; OS, osteosarcoma.

A TUG1 5 '...GAAUUUGGGACUGUUG...3'

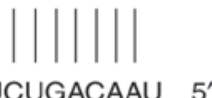

miR-212-3p $\quad 3^{\prime} \ldots$...ACUGACCUCUGACAAU... $5^{\prime}$

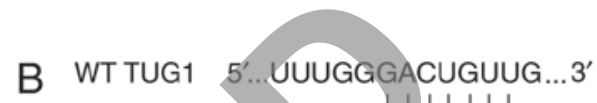

B WT TUG1 5' UUUGGGACUGUUG ...3' miR-212-3p $33^{\prime} . .$. GACCUCUGACAAU ... $5^{\prime}$ MT TUG1 5', UUUGGGAGACAUG ...3'

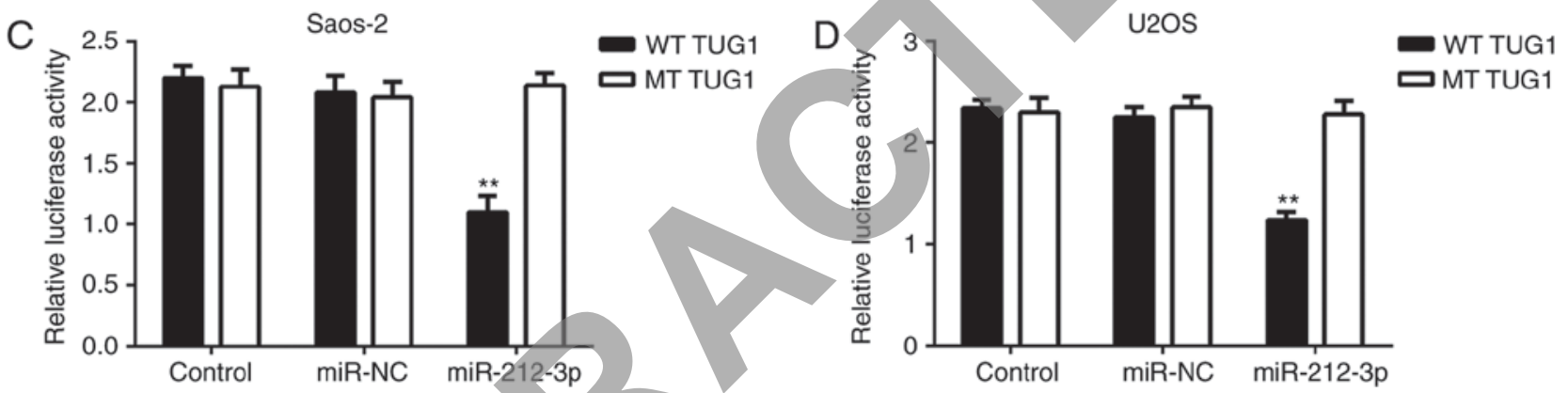

Figure 2. (A) Bioinformatics analysis data indicated the putative binding sites between TUG1 and miR-212-3p. (B) The luciferase reporter plasmid containing the WT or MT miR-212-3p binding sites in TUG1 were constructed. (C) Saos-2 and (D) U2OS cells were used for the luciferase reporter gene assay, and transfection with miR-212-3p mimic significantly inhibited the luciferase activity of WT TUG1 in OS cells, but did not affect the luciferase activity of MT TUG1. ${ }^{* *} \mathrm{P}<0.01$ vs. control. TUG1, taurine upregulated gene 1; miR, microRNA; WT, wild type; MT, mutant type; NC, negative control.

Luciferase reporter gene assay. The fragment from TUG1 containing the wild type (WT) or mutant type (MT) miR-212-3p binding sites were cloned into a pmirGLO Dual-luciferase Target Vector (Promega Corporation, Madison, WI, USA), generating WT and MT TUG1 plasmids. U2OS cells were co-transfected with WT TUG1 plasmid or MT TUG1 plasmid, and miR-212-3p mimic (Sigma-Aldrich; Merck KGaA) or scramble miRNA mimic (Sigma-Aldrich; Merck KGaA) using Lipofectamine 2000 according to the manufacturer's protocol. Following $48 \mathrm{~h}$ for transfection, luciferase reporter gene assay was performed using the Dual-Luciferase Reporter Assay System (Promega Corporation), according to the manufacturer's protocol. The firefly luciferase activities were normalized against Renilla luciferase activity.

Statistical analysis. Data are presented as the mean \pm standard deviation. SPSS 18.0 software (SPSS, Inc., Chicago, IL, USA) was used to perform the statistical analysis. Differences among three or more groups were analyzed with one-way analysis of variance followed by a post-hoc Tukey's test or two-tailed Student's t-test between two groups. $\mathrm{P}<0.05$ was considered to indicate a statistically significant difference.

\section{Results}

TUG1 is upregulated in OS tissues and cell lines. In the present study, the TUG1 expression in OS tissues was examined by performing RT-qPCR. As presented in Fig. 1A, the expression levels of TUG1 were significantly higher in OS tissues compared with adjacent non-tumor tissues. Subsequently, its expression was detected in several common OS cell lines including MG63, Saos-2, U2OS, and HOS, and the normal human osteoblast cell line HFOB1.19 was used as the control group. As indicated in Fig. 1B, the expression levels of TUG1 were also significantly increased in OS cell lines compared with HFOB1.19 cells. Accordingly, TUG1 is upregulated in OS tissues and cell lines. In addition, as Saos-2 and U2OS demonstrated the highest expression levels of TUG1, these two cell lines were used in the following experiments.

TUG1 negatively regulates the expression of $\mathrm{miR}-212-3 p$ by sponging it in OS cells. As lncRNAs can function through interacting with miRNAs, bioinformatics analysis was performed to identify the potential target miRNAs of TUG1 in OS cells. As indicated in Fig. 2A, the putative binding sites between TUG1 and miR-212-3p were identified. To confirm 

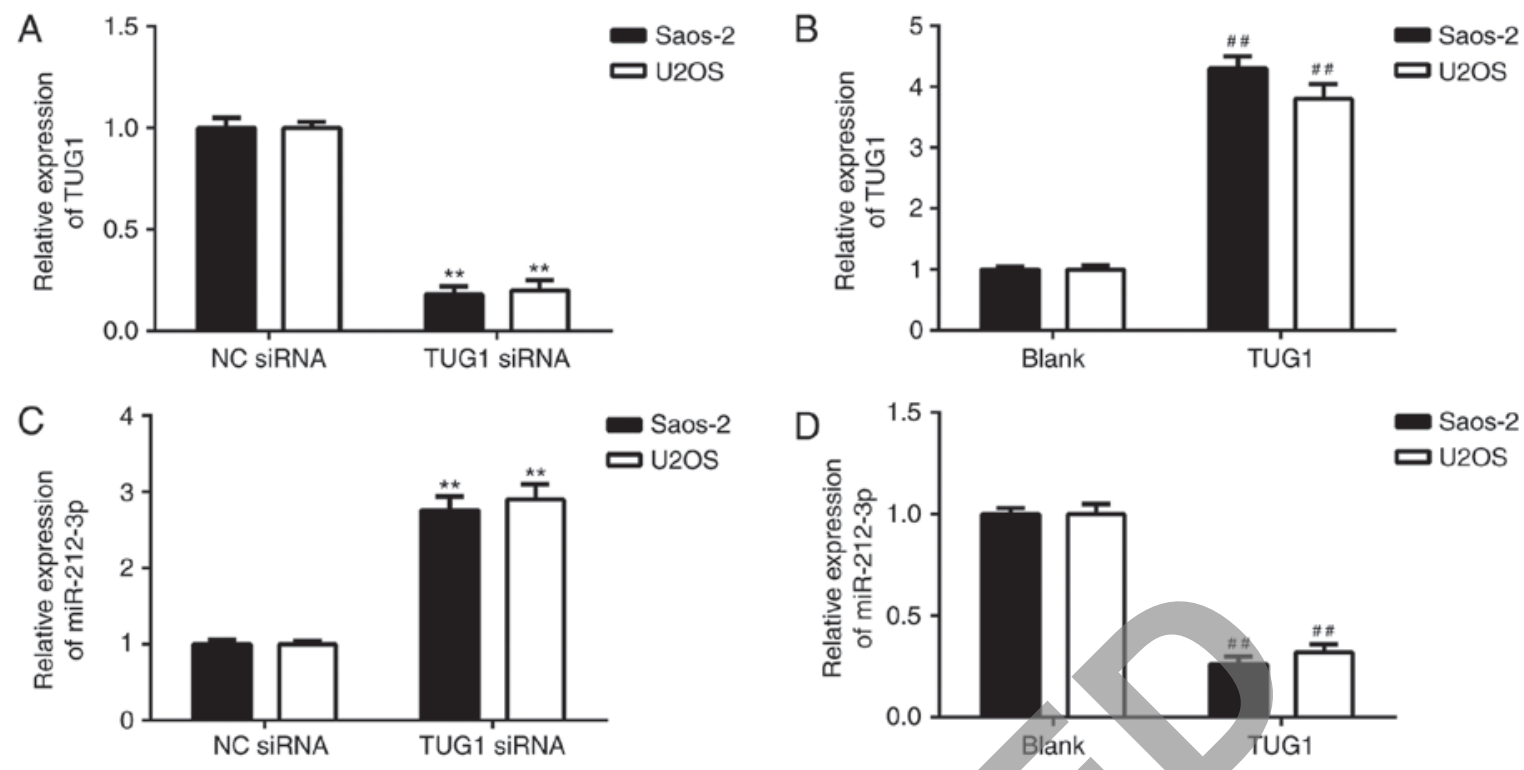

Figure 3. Saos-2 and U2OS were transfected with TUG1 siRNA or TUG1 plasmid to knockdown or upregulate its expression. (A and B) Following transfection, RT-qPCR was conducted to examine the expression of TUG1. (C and D) RT-qPCR was conducted to examine the expression of miR-212-3p. * P $<0.01$ vs. NC siRNA; "\# $\mathrm{P}<0.01$ vs. blank. TUG1, taurine upregulated gene 1; siRNA, short interfering RNA; RT-qPCR, reverse transcription-quantitative polymerase chain reaction; miR, microRNA; NC, negative control.

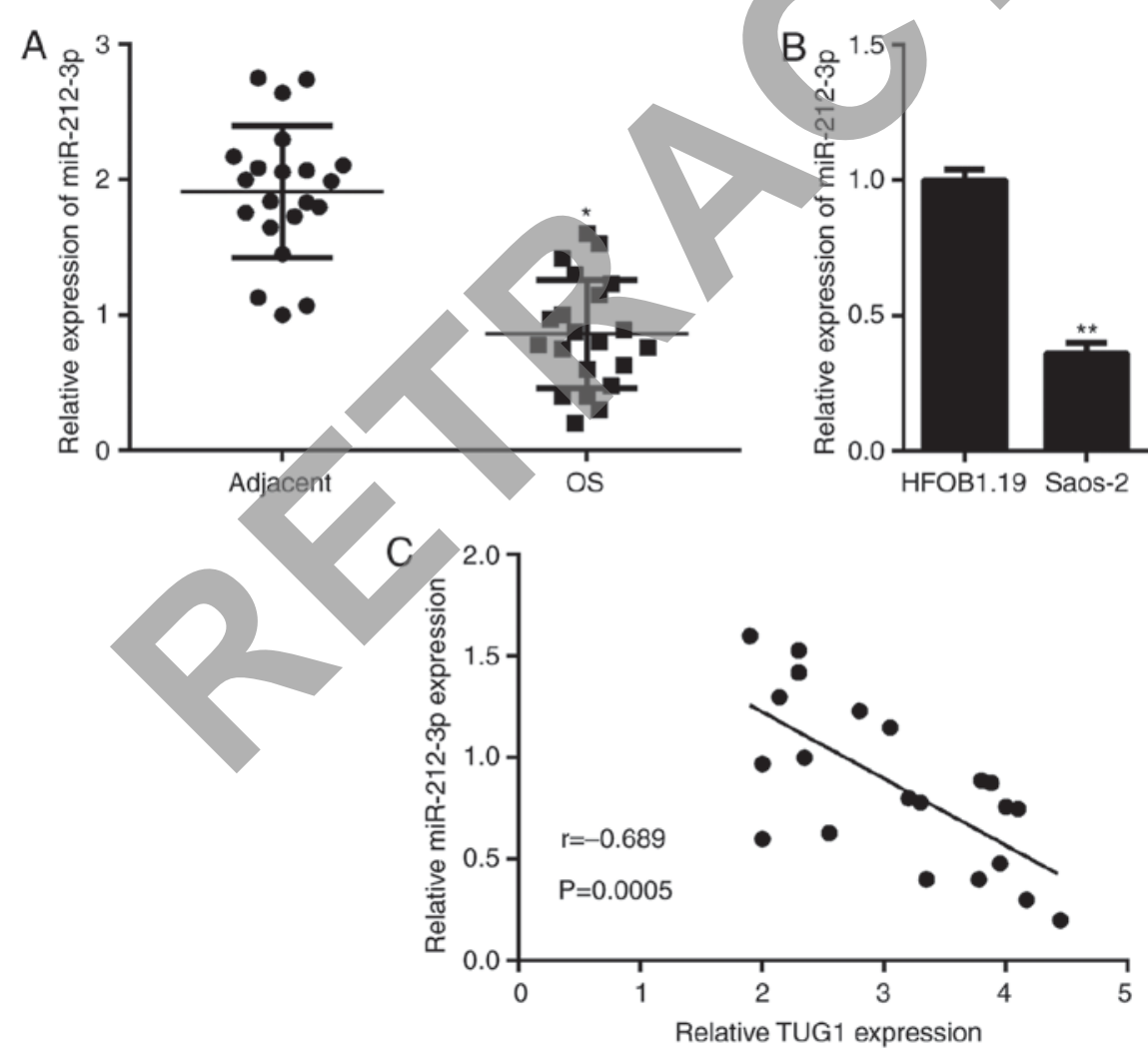

Figure 4. (A) RT-qPCR was conducted to examine the expression of miR-212-3p in OS tissues compared with adjacent non-tumor tissues. "P<0.05 vs. adjacent. (B) RT-qPCR was conducted to examine the expression of miR-212-3p in OS cell lines compared with normal human osteoblast cell line HFOB1.19. ${ }^{* *} \mathrm{P}<0.01$ vs. HFOB1.19. (C) An inverse association between the TUG1 and miR-212-3p expression was observed in OS tissues. RT-qPCR, reverse transcription-quantitative polymerase chain reaction; miR, microRNA; OS, osteosarcoma; TUG1, taurine upregulated gene 1.

this prediction, luciferase reporter plasmid containing the WT or MT miR-212-3p binding sites in TUG1 were constructed (Fig. 2B). As presented in Fig. 2C and D, transfection with miR-212-3p mimic significantly inhibited the luciferase activity of WT TUG1 in Saos-2 and U2OS cells, but did not affect the luciferase activity of MT TUG1. These findings suggest that TUG1 is able to sponge miR-212-3p in OS cells.

The effects of TUG1 on the expression of miR-212-3p were evaluated. Saos-2 and U2OS cells were transfected with TUG1 siRNA or TUG1 plasmid to knockdown or upregulate 

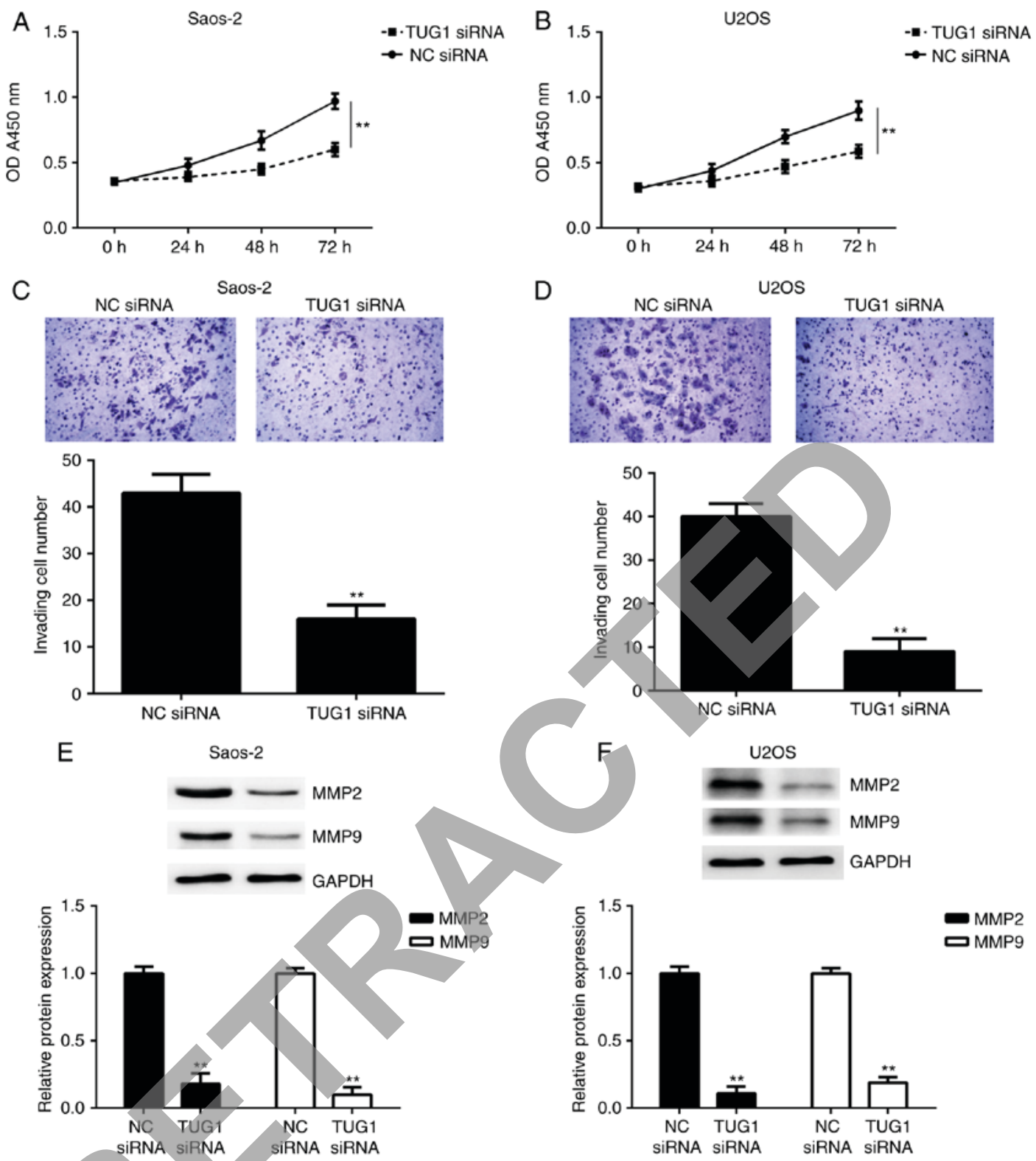

Figure 5. Saos-2 and U2OS were transfected with TUG1 siRNA to knockdown its expression. (A and B) Following transfection, a cell counting kit-8 assay was conducted to examine cell proliferation. ${ }^{* *} \mathrm{P}<0.01$. (C and D) A Transwell assay was used to evaluate cell invasion. (E and F) Western blotting was used to examine the protein expression of MMP2 and MMP9. ${ }^{* *} \mathrm{P}<0.01$ vs. NC siRNA. TUG1, taurine upregulated gene 1; siRNA, short interfering RNA; MMP, matrix metalloproteinase; $\mathrm{NC}$, hegative control; OD, optical density.

its expression, respectively. Following transfection, the expression of TUG1 was significantly decreased in the TUG1 siRNA group compared with NC siRNA group, and significantly increased in the TUG1 group compared with the blank group (Fig. 3A and B). It was also demonstrated that knockdown of TUG1 enhanced miR-212-3p expression (Fig. 3C) and overexpression of TUG1 significantly inhibited expression of miR-212-3p (Fig. 3D) in OS cells. These findings suggest that TUG1 negatively regulates the expression of miR-212-3p by sponging it in OS cells.

miR-212-3p is downregulated in OS. The expression of miR-212-3p was evaluated in OS tissues and cell lines using RT-qPCR. The data indicated that miR-212-3p was significantly downregulated in OS tissues and cell lines, when compared with adjacent non-tumor tissues and normal osteoblasts cell line, respectively (Fig. 4A and B). Furthermore, an inverse association between TUG1 and miR-212-3p expression was observed in OS tissues (Fig. 4C).

Knockdown of TUG1 inhibits OS cell proliferation and invasion. As TUG1 was upregulated in OS, OS cells were transfected with TUG1 siRNA to downregulate its expression, and the effects of TUG1 downregulation on OS cell proliferation and invasion were investigated. A CCK-8 assay was performed to examine cell proliferation. As presented in Fig. 5A and B, the proliferation of Saos-2 and U2OS cells was significantly reduced in the TUG1 siRNA group compared with the NC siRNA group. Following that, a Transwell assay was performed to evaluate cell invasion. As presented in Fig. 5C and D, the invasion of Saos-2 and U2OS cells in the TUG1 siRNA group was significantly 

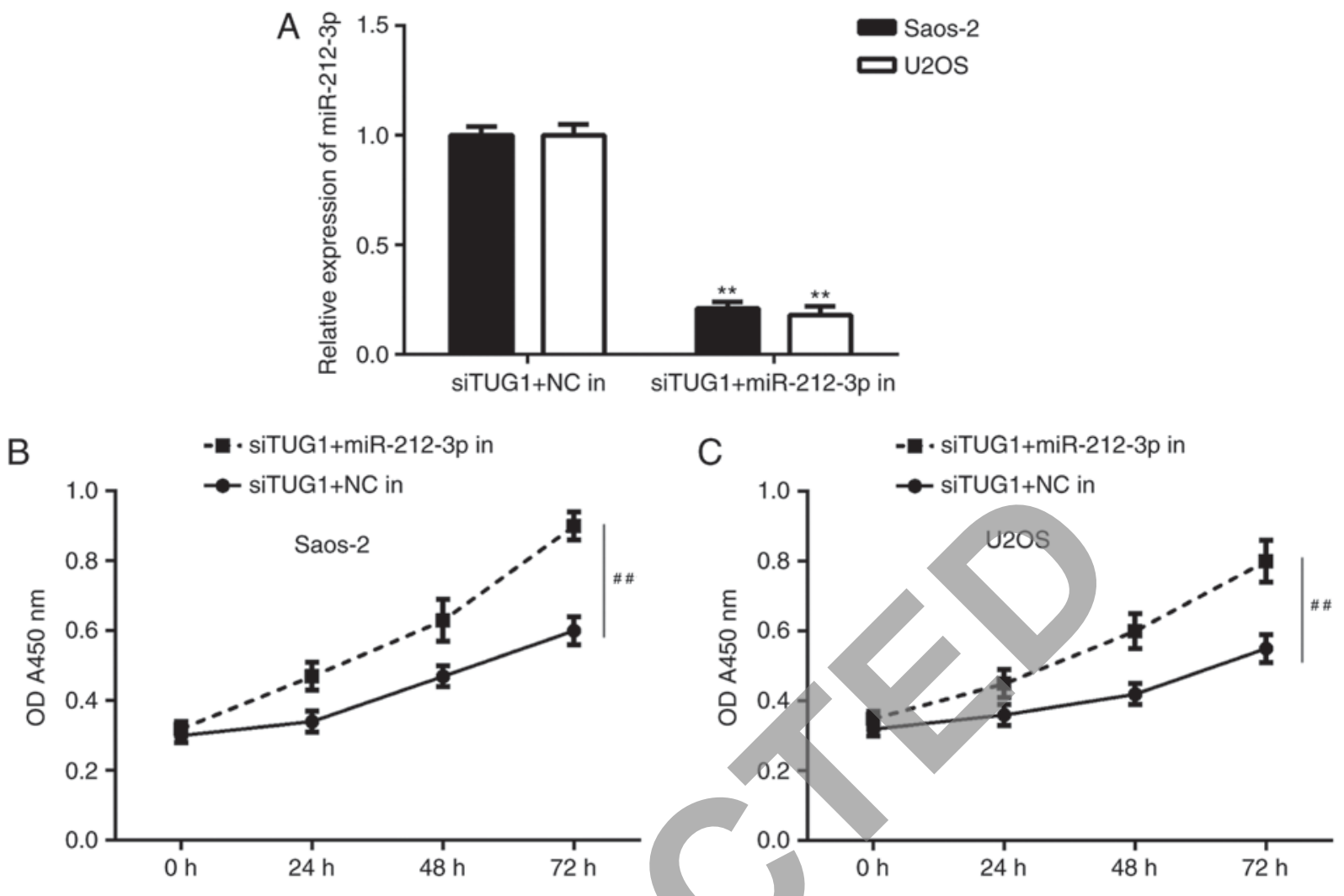

Figure 6. The TUG1 siRNA transfected Saos-2 and U2OS cells were then transfected with NC in or miR-212-3p in, respectively. (A) Following transfection, reverse transcription-quantitative polymerase chain reaction was conducted to examine the expression of miR-212-3p. (B and C) A cell counting kit-8 assay was conducted to examine cell proliferation. ${ }^{* *} \mathrm{P}<0.01$ vs. siTUG1+NC in; ${ }^{\# /} \mathrm{P}<0.01$. TUG1, taurine upregulated gene 1; siRNA, short interfering RNA; NC, negative control; miR, microRNA; in, inhibitor.

inhibited following inhibition of TUGl expression. Consistently, western blot data indicated that the protein levels of MMP2 and MMP9, two key enzymes associated with tumor cell invasion (27), were significantly reduced in the TUG1 siRNA group compared with the NC siRNA group (Fig. 5E and F). Taken together, these findings indicate that knockdown of TUG1 expression inhibits OS cell proliferation and invasion.

Inhibition of TUG1 suppresses OS cell proliferation and invasion by sponging miR-212-3p. Based on the above findings, it was speculated that TUG1 may regulate OS cell proliferation and invasion by sponging miR-212-3p. To clarify this speculation, the TUG1 siRNA transfected OS cells were transfected with NC inhibitor or miR-212-3p inhibitor. Following transfection, miR-212-3p levels were significantly reduced in the TUG1 siRNA+miR-212-3p inhibitor group compared with the TUG1 siRNA+NC inhibitor group (Fig. 6A). Further investigation indicated that the proliferation of OS cells was significantly increased in the TUG1 siRNA+miR-212-3p inhibitor group compared with the TUG1 siRNA+NC inhibitor group (Fig. 6B and C).

Similarly, the cell invasion capacity was also significantly higher in the TUG1 siRNA+miR-212-3p inhibitor group compared with the TUG1 siRNA+NC inhibitor group (Fig. 7A and B). Furthermore, the suppressive effects of TUG1 inhibition on the protein expression of MMP2 and MMP9 were significantly ameliorated following co-transfection with miR-212-3p inhibitor (Fig. 7C-D). Accordingly, these findings demonstrate that Inhibition of TUG1 suppresses OS cell proliferation and invasion by sponging miR-212-3p.

\section{Discussion}

The molecular mechanism of TUG1 underlying OS growth and metastasis remains largely unclear. In the present study, it was demonstrated that TUG1 was significantly upregulated whereas miR-212-3p was significantly downregulated in OS tissues and cell lines, when compared with adjacent non-tumor tissues and normal osteoblast cell lines, respectively. An inverse association between the TUG1 and miR-212-3p expression was also observed in OS tissues. Furthermore, TUG1 directly interacted with miR-212-3p and negatively regulated the expression of miR-212-3p in OS cells. In vitro experiments further indicated that inhibition of TUG1 suppressed the proliferation and invasion of OS cells. Furthermore, knockdown of miR-212-3p eliminated the suppressive effects of TUG1 inhibition on the proliferation and invasion of OS cells.

In recent years, TUG1 has been demonstrated to be aberrantly upregulated in certain common human cancers, and has been suggested to serve a promoting role during tumor progression $(15,17,28)$. For instance, TUG1 is significantly upregulated in gastric cancer tissues and significantly correlated with clinicopathological characteristics (29). $\mathrm{Xu}$ et al (30) recently reported an upregulation of TUG1 in both cholangiocarcinoma tissues and cell lines, and that its overexpression is linked to tumor size, TNM stage, postoperative recurrence and overall survival of cholangiocarcinoma 
A
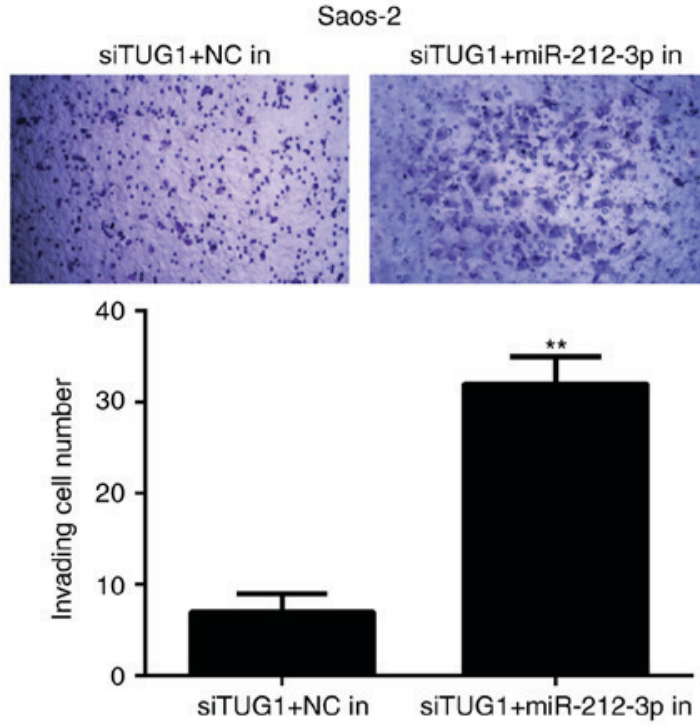

C

aos-2

Saos-2
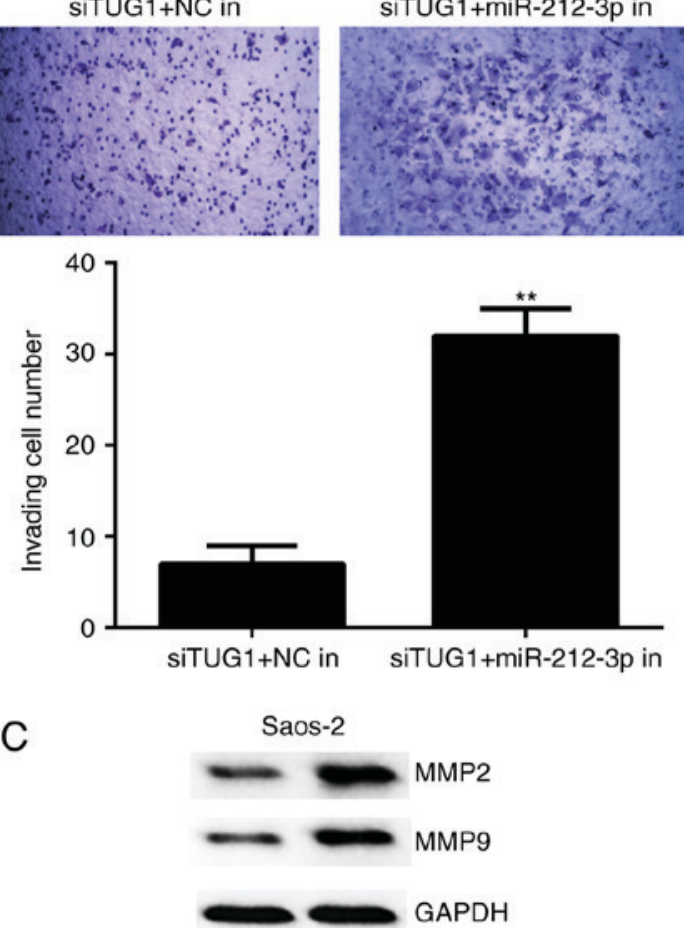

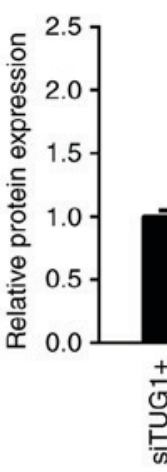

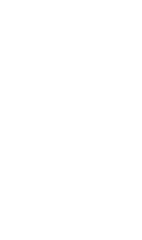

B
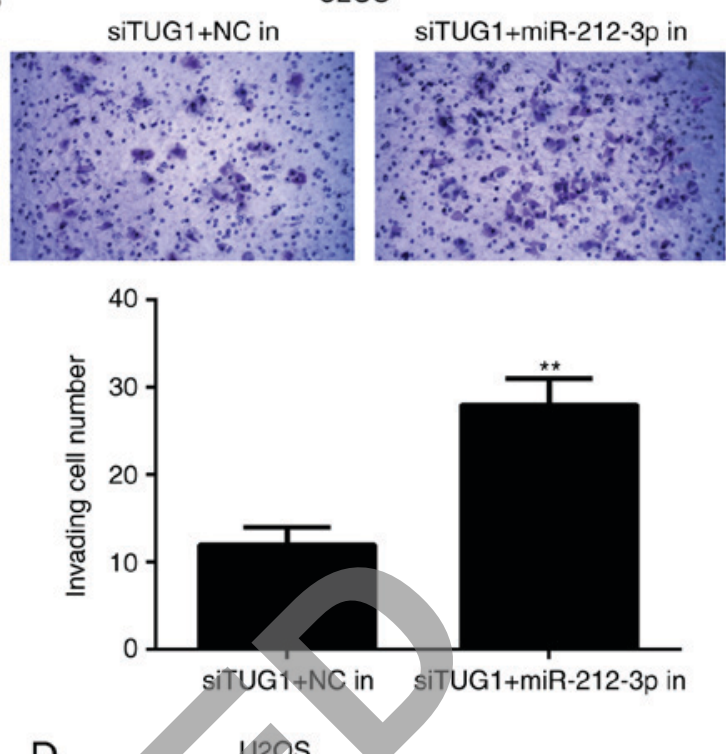

D

D U20s
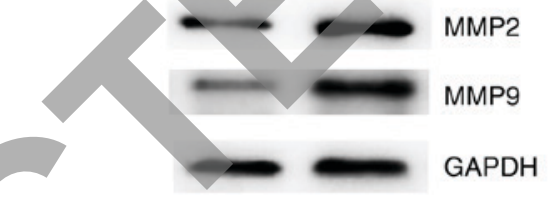

MMP2
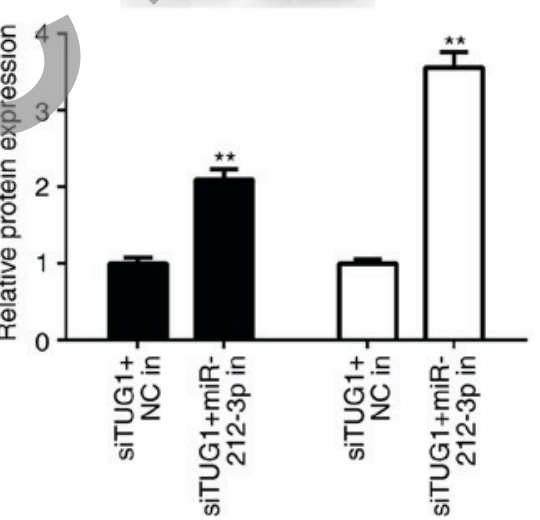

MMP2

صMP9

Figure 7. The TUG1 siRNA transfected Saos-2 and U2OS cells were then transfected with NC inhibitor or miR-212-3p in, respectively. (A and B) Following transfection, a Transwell assay was used to study cell invasion. (C and D) Western blotting was used to examine the protein expression of MMP2 and MMP9.

${ }^{* *} \mathrm{P}<0.01$ vs. siTUG1+NC in. TUG1, taurine upregulated gene 1; siRNA, short interfering RNA; NC, negative control; miR, microRNA; in, inhibitor; MMP, matrix metalloproteinase.

patients. Furthermore, knockdown of TUG1 inhibited cholangiocarcinoma cell growth and metastasis in vitro by inhibition of epithelial-mesenchymal transition (EMT) (30). In addition, TUG1 promotes papillary thyroid cancer cell proliferation, migration and EMT formation through targeting miR-145 (31). In the present study, it was also demonstrated that the expression of TUG1 was significantly upregulated in OS tissues and cells, consistent with previous studies $(18,32)$. Furthermore, silencing of TUG1 by siRNA caused a significant decrease in OS cell proliferation and invasion in vitro.

Furthermore, IncRNAs have been demonstrated to act as endogenous sponges of miRNAs, and negatively regulate the expression of miRNAs through sponging them $(28,33)$. For instance, TUG1 promotes cell proliferation and metastasis by negatively regulating miR-300 in gallbladder carcinoma (28). Furthermore, TUG1 promotes endometrial cancer development via inhibiting miR-299 and miR-34a-5p (33). In OS,
TUG1 contributes to tumorigenesis by sponging miR-9-5p and regulating POU2F1 expression (19). Wang et al (20) recently demonstrated that TUG1 promoted OS cell migration and invasion by acting as a ceRNA of miR-335-5p. Cao et al (22) also reported that TUG1 promoted OS tumorigenesis by upregulating EZH2 expression via miR-144-3p. In addition, knockdown of TUG1 inhibits the proliferation and invasion of OS cells by sponging miR-153 (21). In the present study, bioinformatics analysis and luciferase reporter assay confirmed that TUG1 is an endogenous sponge of miR-212-3p, and the expression of miR-212-3p was negatively regulated by TUG1 in OS cells in vitro. Furthermore, it was demonstrated that the miR-212-3p levels were significantly reduced in OS tissues and cell lines, when compared with adjacent non-tumor tissues and normal osteoblasts cell line, respectively, and an inverse association between TUG1 and miR-212-3p expression was observed in OS tissues. Therefore, these findings suggest 
that the increased expression of TUG1 may contribute to the reduced miR-212-3p in OS.

The suppressive role of miR-212 has previously been reported in OS $(24,25)$. miR-212 inhibits OS cells proliferation and invasion by directly targeting SOX4 and FOXA1 $(24,25)$. However, the regulatory mechanism of miR-212-3p expression in OS cells still remains largely unclear. In the present study, it was demonstrated that knockdown of TUG1 inhibited OS cell proliferation and invasion, whereas these effects were rescued when the expression of miR-212-3p was suppressed. These findings suggest that inhibition of TUG1 suppresses OS cell proliferation and invasion by sponging miR-212-3p.

In summary, the present study demonstrates that TUG1 serves a promoting role in regulating OS cell proliferation and invasion by sponging miR-212-5p, and thus expands the knowledge of the molecular mechanisms of OS. However, the present study is limited by the small number of clinical samples utilized. Future studies should also utilize animals in order to further results.

\section{Acknowledgements}

Not applicable.

\section{Funding}

No funding was received.

\section{Availability of data and materials}

All data generated or analyzed during this study are included in this published article.

\section{Authors' contributions}

HL wrote the manuscript and performed the experiments; GT collected clinical samples; FT performed statistical analysis; and LS designed the present study and revised the manuscript.

\section{Ethics approval and consent to participate}

The present study was approved by the Medical Ethics Committee of Daqing Longnan Hospital (Daqing, China). All patients provided written informed consent.

\section{Consent for publication}

All patients provided written informed consent.

\section{Competing interests}

The authors declare that they have no competing interests.

\section{References}

1. Vander Griend RA: Osteosarcoma and its variants. Orthop Clin North Am 27: 575-581, 1996.

2. Lindsey BA, Markel JE and Kleinerman ES: Osteosarcoma overview. Rheumatol Ther 4: 25-43, 2017.

3. Siegel RL, Miller KD and Jemal A: Cancer statistics, 2015. CA Cancer J Clin 65: 5-29, 2015.
4. Simpson S, Dunning MD, de Brot S, Grau-Roma L, Mongan NP and Rutland CS: Comparative review of human and canine osteosarcoma: Morphology, epidemiology, prognosis, treatment and genetics. Acta Vet Scand 59: 71, 2017.

5. Wang S, Hui Y, Li X and Jia Q: Silencing of lncRNA-CCDC26 restrains the growth and migration of glioma cells in vitro and in vivo via targeting miR-203. Oncol Res: Jun 9, 2017 (Epub ahead of print).

6. Li J, Zi Y, Wang W and Li Y: Long noncoding RNA MEG3 inhibits cell proliferation and metastasis in chronic myeloid leukemia via targeting miR-184. Oncol Res 26: 297-305, 2018.

7. Zhu K, He Y, Xia C, Yan J, Hou J, Kong D, Yang Y and Zheng G: MicroRNA-15a inhibits proliferation and induces apoptosis in CNE1 nasopharyngeal carcinoma cells. Oncol Res 24: 145-151, 2016.

8. Zhou Y, Yang C, Wang K, Liu X and Liu Q: MicroRNA-33b inhibits the proliferation and migration of osteosarcoma cells via targeting hypoxia-inducible factor-1 $\alpha$. Oncol Res 25: 397-405, 2017.

9. Zhang JJ, Wang DD, Du CX and Wang Y: Long noncoding RNA ANRIL promotes cervical cancer development by acting as a sponge of miR-186. Oncol Res: May 22, 2017 (Epub ahead of print).

10. Yang M,Zhai X, Ge T, Yang C and Lou G: MiR-181a-5p promotes proliferation and invasion and inhibits apoptosis of cervical cancer cells via regulating inositol polyphosphate-5-phosphatase A (INPP5A). Oncol Res: Jun 23, 2017 (Epub ahead of print).

11. Wang Y, Li J, Xu C and Zhang X: MicroRNA-139-5p inhibit cell proliferation and invasion by targeting RHO-associated coiled-coil containing protein kinase 2 in ovarian cancer. Oncol Res: Jun 14, 2017 (Epub ahead of print)

12. Wang C, Zhou B, Liu M, Liu Y and Gao R: miR-126-5p restoration promotes cell apoptosis in cervical cancer by targeting Bcl212. Oncol Res 25: 463-470, 2017.

13. Li Z, Guo J, Ma Y, Zhang L and Lin Z: Oncogenic role of MicroRNA-30b-5p in glioblastoma through targeting proline-rich transmembrane protein 2. Oncol Res 26: 219-230, 2018.

4. Hua F, Li CH, Chen XG and Liu XP: Long noncoding RNA CCAT2 knockdown suppresses tumorous progression by sponging miR-424 in epithelial ovarian cancer. Oncol Res 26: 241-247, 2018.

15. Zhang Q, Geng PL, Yin P, Wang XL, Jia JP and Yao J: Down-regulation of long non-coding RNA TUG1 inhibits osteosarcoma cell proliferation and promotes apoptosis. Asian Pac J Cancer Prev 14: 2311-2315, 2013.

16. Zhu J, Shi H, Liu H, Wang X and Li F: Long non-coding RNA TUG1 promotes cervical cancer progression by regulating the miR-138-5p-SIRT1 axis. Oncotarget 8: 65253-65264, 2017.

17. Yan G, Wang X, Yang M, Lu L and Zhou Q: Long non-coding RNA TUG1 promotes progression of oral squamous cell carcinoma through upregulating FMNL2 by sponging miR-219. Am J Cancer Res 7: 1899-1912, 2017.

18. Ma B, Li M, Zhang L, Huang M, Lei JB, Fu GH, Liu CX, Lai QW, Chen QQ and Wang YL: Upregulation of long non-coding RNA TUG1 correlates with poor prognosis and disease status in osteosarcoma. Tumour Biol 37: 4445-4455, 2016.

19. Xie CH, Cao YM, Huang Y, Shi QW, Guo JH, Fan ZW, Li JG, Chen BW and Wu BY: Long non-coding RNA TUG1 contributes to tumorigenesis of human osteosarcoma by sponging miR-9-5p and regulating POU2F1 expression. Tumour Biol 37: 15031-15041, 2016.

20. Wang Y, Yang T, Zhang Z, Lu M, Zhao W, Zeng X and Zhang W: Long non-coding RNA TUG1 promotes migration and invasion by acting as a ceRNA of miR-335-5p in osteosarcoma cells. Cancer Sci 108: 859-867, 2017.

21. Wang H, Yu Y, Fan S and Luo L: Knockdown of long noncoding RNA TUG1 inhibits the proliferation and cellular invasion of osteosarcoma cells by sponging MiR-153. Oncol Res: Apr 12, 2017 (Epub ahead of print).

22. Cao J, Han X, Qi X, Jin X and Li X: TUG1 promotes osteosarcoma tumorigenesis by upregulating EZH2 expression via miR-144-3p. Int J Oncol 51: 1115-1123, 2017.

23. Wada R, Akiyama Y, Hashimoto Y, Fukamachi H and Yuasa Y: miR-212 is downregulated and suppresses methyl-CpG-binding protein $\mathrm{MeCP} 2$ in human gastric cancer. Int J Cancer 127: 1106-1114, 2010.

24. Luo XJ, Tang DG, Gao TL, Zhang YL, Wang M, Quan ZX and Chen J: MicroRNA-212 inhibits osteosarcoma cells proliferation and invasion by down-regulation of Sox4. Cell Physiol Biochem 34: 2180-2188, 2014. 
25. Liu J, Chen B, Yue B and Yang J: MicroRNA-212 suppresses the proliferation and migration of osteosarcoma cells by targeting forkhead box protein A1. Exp Ther Med 12: 4135-4141, 2016.

26. Livak KJ and Schmittgen TD: Analysis of relative gene expression data using real-time quantitative PCR and the 2(-Delta Delta C(T)) method. Methods 25: 402-408, 2001

27. Braicu EI, Gasimli K, Richter R, Nassir M, Kümmel S, Blohmer JU, Yalcinkaya I, Chekerov R, Ignat I, Ionescu A, et al: Role of serum VEGFA, TIMP2, MMP2 and MMP9 in monitoring response to adjuvant radiochemotherapy in patients with primary cervical cancer-results of a companion protocol of the randomized NOGGO-AGO phase III clinical trial. Anticancer Res 34: 385-391, 2014.

28. Ma F, Wang SH, Cai Q, Jin LY, Zhou D, Ding J and Quan ZW: Long non-coding RNA TUG1 promotes cell proliferation and metastasis by negatively regulating miR-300 in gallbladder carcinoma. Biomed Pharmacother 88: 863-869, 2017.

29. Baratieh Z, Khalaj Z, Honardoost MA, Emadi-Baygi M, Khanahmad H, Salehi M and Nikpour P: Aberrant expression of PlncRNA-1 and TUG1: Potential biomarkers for gastric cancer diagnosis and clinically monitoring cancer progression. Biomark Med 11: 1077-1090, 2017.
30. Xu Y, Leng K, Li Z, Zhang F, Zhong X, Kang P, Jiang X and Cui Y: The prognostic potential and carcinogenesis of long non-coding RNA TUG1 in human cholangiocarcinoma. Oncotarget 8: 65823-65835, 2017.

31. Lei H, Gao Y and Xu X: LncRNA TUG1 influences papillary thyroid cancer cell proliferation, migration and EMT formation through targeting miR-145. Acta Biochim Biophys Sin (Shanghai) 49: 588-597, 2017.

32. Yun-Bo F, Xiao-Po L, Xiao-Li L, Guo-Long C, Pei Z and Fa-Ming T: LncRNA TUG1 is upregulated and promotes cell proliferation in osteosarcoma. Open Med (Wars) 11: 163-167, 2016.

33. Liu L, Chen X, Zhang Y, Hu Y, Shen X and Zhu W: Long non-coding RNA TUG1 promotes endometrial cancer development via inhibiting miR-299 and miR-34a-5p. Oncotarget 8: 31386-31394, 2017

cc) (i) () Attribution-NonCommercial-NoDerivatives 4.0 International (CC BY-NC-ND 4.0) License. 